\title{
Relación entre dieta mediterránea, actividad física e índice de masa corporal en adolescentes de secundaria de dos pueblos de Granada
}

\section{Relationship between mediterranean diet, physical activity and body mass index in adolescent of high school of two villages in Granada}

\author{
Eva Ma Peláez-Barrios*, Mercedes Vernetta-Santana y Jesús López-Bedoya
}

Departamento de Educación Física y Deporte. Facultad de Ciencias de la Actividad Física y el Deporte. Universidad de Granada, España

\begin{abstract}
Resumen: El objetivo fue evaluar la Adherencia a la Dieta Mediterránea (ADM) en adolescentes y su relación con el Índice de Masa Corporal (IMC) y Actividad Física (AF). Participaron 209 estudiantes (117 chicas y 92 chicos) entre 12 y 18 ańos. Se analizó la ADM a través del test KIDMED y el grado de AF se estimó mediante el Cuestionario Internacional de Actividad Física. Se midió la altura y el peso de cada adolescente, calculándose el IMC. El 65,6\%, de la muestra total, mostró una óptima ADM con un IMC de $20,02 \mathrm{~kg} / \mathrm{m} 2$ sin diferencias de sexos. Sin embargo, los chicos mostraron mayor nivel de AF que las chicas, con diferencias estadísticamente significativas. No se encontraron relaciones significativas entre las variables DM, AF, IMC y género. Los hábitos de los adolescentes espańoles de la muestra son saludables, presentando la mayoría óptima ADM, valores normales del IMC y buen nivel de AF.

Palabras clave: Test Kidmed, índice de masa corporal; Actividad Física, adolescentes, Cuestionario Internacional de Actividad Física (IPAQ).
\end{abstract}

Abstract: The objetive was evaluate the Adherence to Mediterranean Diet (AMD) in adolescents and the relationship with the Body Mass Index (BMI) and Physical Activity (PA). The study was realized with 209 adolescents (117 girls y 92 boys), between 12 and 18 years. The AMD was analysed through the KIDMED test and PA level was estimated using the International Physical Activity Questionnaire (IPAQ short version. The height and weigth was measured of each gymnastics, calculating BMI. The $65,6 \%$, of the total sample showed an optimal AMD with a BMI of 20.02 $\mathrm{kg} / \mathrm{m} 2$ without sex differences. However, the boys showed significantly bigger physical activity level than girls. No significant relationships were found between variables MD, PA, BMI and gender. The habits of the Spanish adolescents in the sample are healthy, presenting the most optimal AMD, normal values of BMI and in good level of PA.

Key Words: KidMed test, body mass index; Physical activity; adolescents, International Physical Activity questionaire.

\section{Introducción}

La adolescencia es considerado un periodo de transición entre la infancia y la edad adulta que se caracteriza sobre todo por los numerosos cambios tanto físicos como psicológicos, sociales e intelectuales (HealthyChildren.org, 2017). En esta etapa la falta de hábitos saludables, las dietas poco variadas y equilibradas, así como la falta de Actividad Física (AF) están a la orden del día y van asociadas a un riesgo para la salud futura de los niños/as y adolescentes, por tanto, desde la infancia deberían de inculcarse hábitos de dietas saludables y AF para prevenir las enfermedades que dichos hábitos puedes acarrear (García, Garrido \& Ojembarrena, 2008).

Varios estudios realizados, no sólo en España sino en otras partes del mundo, han indicado que los españoles suelen tener una buena alimentación en comparación con los adolescentes de otras culturas (Acosta, Llopis, Gómez \& Pineda, 2005). Sin embargo, existen numerosas investigaciones que indican que a pesar de este positivo dato, existe un alto porcentaje de la población en posesión de enfermedades cardiovasculares

Dirección para correspondencia [Correspodence address]: Eva Ma Peláez Barrios. Departamento de Educación Física y Deporte. Facultad de Ciencias de la Actividad Física y el Deporte. Universidad de Granada (España).E-mail: evapelaezbarrios@gmail.com como el sobrepeso y la obesidad (Muñoz, 2005; SánchezCruz, Jiménez-Moleón, Fernández-Quesada \& Sánchez, 2013). Dichos estudios indican que este alto porcentaje es debido al sedentarismo de la población junto con los malos hábitos alimentarios que poseen, aunque este porcentaje no ha incrementado en los últimos años (Sánchez-Cruz, Jiménez-Moleón, Fernández-Quesada \& Sánchez, 2012).

La Dieta Mediterránea (DM) es uno de los modelos dietéticos más saludables con beneficios frente a estas enfermedades comentadas anteriormente y como consecuencia, una mayor expectativa de vida (Martínez-González et al., 2008). Se caracteriza por una dieta basada fundamentalmente en los alimentos típicos de la región mediterránea, de ahí su denominación. La adhesión a esta dieta, se cuantifica de forma rápida con el Cuestionario de Calidad de la Dieta Mediterránea en la Infancia y la adolescencia: KIDMED (García, Herrera, Rodríguez, Nissensohn, Román-Viñas \& SerraMajem, 2015).

Por otro lado, la AF es también un importante factor de hábitos de vida saludable (Balaguer, 2004), siendo numeroso los estudios que recogen los beneficios positivos de la práctica de AF sobre la salud física y psicológica (Labrado, 2011). Sin embargo, se acepta la idea que el porcentaje de práctica de AF 
disminuye con respecto a la infancia, de modo que un $40,8 \%$ de los adolescentes españoles no realiza ningún tipo de ejercicio (Tercedor, Martín-Matillas, Chillón, Pérez-López, Ortega \& Wärnberg, 2007). Por ello, la Fundación Española de Nutrición (FEN) indica que España es uno de los países de la Unión Europea con los índices de sedentarismo más elevado (Valera-Moreinas, 2013).

En la bibliografía revisada, aparecen muchos trabajos sobre la adherencia a la dieta mediterránea en los adolescentes y por otro lado el tipo de actividad de forma independiente. Sin embargo, son pocos los que estudian la combinación de hábitos dietéticos saludables junto con la práctica regular de ejercicio físico (Doménech, Sánchez \& Ros, 2015; Grao-Cruces, Nuviala, Fernandez-Martinez, Porcel-Galvez, Moral-Garcia \& Martinez-Lopez, 2013).

De ahí que el objetivo de este estudio fue evaluar la adherencia a la dieta mediterránea en adolescentes de primer y segundo ciclo de la ESO y su relación con el IMC y Actividad Física.

\section{Material y métodos}

\section{Participantes}

En el presente estudio participaron 209 estudiante (117 chicas y 92 chicos), entre 12 y 18 años. La muestra se distribuyó en dos grupos en función del ciclo escolar: el primer ciclo de secundaria, de 12 a 14 años estaba formado por 68 chicas $(53,5 \%)$ y 54 chicos $(45 \%)$ y el segundo ciclo de secundaria 14 a16 años estaba formado por 49 chicas $(38,6 \%)$ y 38 chi$\cos (31,7 \%)$. Todos participaron de forma voluntaria, tras solicitar el consentimiento informado a los padres o tutores en función de la legislación vigente (Ley 41/2002 de 14 de noviembre) y se respetó los principios éticos expresados en la Declaración de Helsinki.

\section{Diseño e Instrumentos}

Se realizó un estudio observacional, descriptivo, transversal y correlacional. Los instrumentos utilizados para las mediciones de las distintas variables fueron:

a) Variables antropométricas. El peso se determinó con una báscula digital TEFAL, precisión de $0,05 \mathrm{~kg}$ y para la talla se utilizó un tallímetro SECA 220 con precisión de $1 \mathrm{~mm}$. Con ambas medidas se calculó el Índice de Masa Corporal (IMC), en base al peso (kg) dividido por la altura al cuadrado (metros), es decir, índice de Quetelet $(\mathrm{Kg} / \mathrm{m} 2)$ para conocer el estado de salud en el que se encontraban los adolescentes. Al ser adolescentes, se utilizó los indicadores propuesto por Cole, Flegal, Nicholls \& Jackson (2007) delgadez grado III $(<16)$; delgadez grado II (16,1 a 17); delgadez grado I
(17,1 a 18,5$)$; normal $(18,5$ a 24,9$)$, sobrepeso (25 a 30); y obesidad $(\geq 30)$.

b) Adherencia a la Dieta mediterránea. La calidad de dieta de los adolescentes se obtuvo de acuerdo al índice KIDMED, conocido también como Test de calidad de la Dieta Mediterránea. El índice puede oscilar entre 0 y 12 , y se basa en un test de 16 preguntas relacionadas con su alimentación. Aquellas preguntas que incluyen una respuesta negativa en relación con la DM valen -1 y las que aportan un aspecto positivo +1 punto. La suma de los valores se califica en 3 niveles según la adherencia que posea a la DM: $\geq 8=$ óptima, 4-7 = medio $y \leq 3=$ baja (Serra et al. 2004 et al. 2010).

c) Grado de Actividad Física. Para conocer el nivel de práctica de AF fuera del horario escolar que tienen los alumnos se utilizó el cuestionario IPAQ (versión corta), por ser un instrumento válido y fiable en las edades estudiadas, el cual consta de 7 ítems que mide la actividad vigorosa, moderada y caminatas que realiza el alumno así como la inclusión de distintas preguntas que nos relatan la frecuencia (media en días por semana) y duración (tiempo por día) en cada tipo de actividad. Los resultados de este cuestionario se haya mediante una medida (equivalentes metabólicos METs) adquiridos con una serie de fórmulas según un protocolo establecido para procesar y analizar dichos datos (htpp://www.ipaq.ki.se). En función a los MET consumidos, el IPAQ establece 3 niveles de AF: baja, moderada y alta (Delgado, Soto \& Tercedor, 2005):

Baja, incluye a aquellos individuos que no realizan actividad o hacen alguna actividad, pero no es suficiente para poder situarlos en los criterios de las categorías moderada y/o alta.

Moderada, cuando se cumple con alguno de los siguientes criterios: tres días o más de actividad física de intensidad vigorosa al menos 20 min por día, o cinco o más días de intensidad física moderada y/o andar al menos $30 \mathrm{~min}$ por día, o cinco o más días de cualquier combinación de andar, actividad de intensidad moderada y actividad de intensidad vigorosa sumando un mínimo Total de actividad Física de al menos $600 \mathrm{MET}-\mathrm{min} / \mathrm{sem}$.

Alta, se considera si cumple con cualquiera de los criterios siguientes: actividades de intensidad vigorosa al menos tres días por semana sumando un mínimo total de actividad física de al menos $1500 \mathrm{MET}-\mathrm{min} / \mathrm{sem}$, o siete o más días de cualquier combinación de andar, intensidad moderada o actividades de intensidad vigorosa sumando un mínimo total de actividad física de al menos $3000 \mathrm{MET}-\mathrm{min} / \mathrm{sem}$. 


\section{Procedimiento}

El estudio se llevó a cabo en dos institutos, el IES Alfaguara (Loja) y el IES Montes Orientales (Iznalloz). Previamente, para la recopilación de datos y mediciones, se contactó con los directores de ambos institutos explicándole el objetivo del estudio y solicitándoles su permiso y colaboración para la realización del mismo. El alumnado participó de forma voluntaria selecionados en la clase de Educación Física (EF), tras explicarle el estudio en presencia de su profesor/a responsable, indicándoles la confidencialidad y el carácter anónimo de sus datos. Todas las medidas se realizaron en período lectivo durante los meses de Noviembre y Diciembre del curso escolar 2016/2017 en su horario lectivo de las clases de EF. Los estudiantes cumplimentaron los cuestionarios KIDMED e IPAQ mediante la supervisión del profesor de EF y una de las autoras de este trabajo garantizando el anonimato de los mismos. Igualmente, durante este horario, se determinó el peso y la talla. En todas las mediciones el adolescente estuvo descalzo y con ropa ligera.

\section{Análisis Estadístico}

Primeramente, se efectuó las pruebas de normalidad y homocedasticidad de las distribuciones a través de los estadístico Kolmogorov Smirnov y Levene respectivamente. Al no observar una distribución normal en parte de las distribuciones de los valores registrados del nivel de Actividad Física e Índice de adherencia a la dieta mediterránea, según Sexo, IMC y Ciclo educativo se optó por un análisis no paramétrico. Las Pruebas de Kruskal Wallis y U de Mann Whitney se realizó para el contraste de muestras independientes,. El tamaño del efecto (r) se calculó aplicando la fórmula $\mathrm{Z} / \sqrt{ } \mathrm{N}$ ( $Z$ entre raíz cuadrada de $\mathrm{N}$ ). Finalmente, el análisis de correlación entre las distintas variables se realizó mediante el estadístico R de Spearman. Los datos se muestran en rangos promedio. Los análisis estadísticos se realizaron utilizando SPSS, versión 22.0 (SPSS Inc., Chicago, IL, USA).

\section{Resultados}

La Tabla 1, muestra las características generales de los estudiantes, según el sexo y ciclo escolar.

Tabla 1. Composición de la muestra

\begin{tabular}{lllll}
\hline & & $1^{\text {er }}$ Ciclo ESO & 20 Ciclo ESO & TOTAL \\
\hline Chicas & $\mathrm{N}$ & 68 & 49 & 117 \\
& Peso (Kg) & $47,55(10,20)$ & $57,00(9,06)$ & $51,5(10,77)$ \\
& Talla (m) & $1,59(, 08)$ & $1,64(, 08)$ & $1,61(, 08)$ \\
& IMC & $18,76(3,09)$ & $21,15(3,04)$ & $19,76(3,28)$ \\
& Edad (años) & $13,01(0,63)$ & $15,37(, 49)$ & $14,00(1,30)$ \\
\hline Chicos & N & 54 & 38 & 92 \\
& Peso (Kg) & $51,88(12,54)$ & $65,07(13,03)$ & $1,67(, 11)$ \\
& Talla (m) & $1,62(, 10)$ & $1,73(, 08)$ & $20,35(3,56)$ \\
& IMC & $19,46(3,41)$ & $21,63(3,42)$ & $14,02(1,30)$ \\
\hline Total & Edad (años) & $13,09(, 78)$ & $15,34(, 48)$ & 209 \\
& N & 122 & 87 & $54,07(12,72)$ \\
& Peso (Kg) & $49,47(11,45)$ & $60,52(11,62)$ & $1,63(, 10)$ \\
& Talla (m) & $1,60(, 09)$ & $1,68(, 09)$ & $20,02(3,41)$ \\
& IMC & $19,07(3,24)$ & $21,36(3,20)$ & $14,01(1,30)$ \\
\hline
\end{tabular}

Los datos se muestran en valores promedio (desviación estándar)

En relación al Índice de Adherencia a la DM, el 65,6\% de la muestra total obtuvo una puntuación buena $(\geq 8)$, el $34 \%$ una puntuación media (4-7) y sólo el $0,5 \%$ pobre $(\leq 3)$ (Tabla 2$)$. No se encontró diferencias significativas entre chicos y chicas en los porcentajes de buena adherencia a la DM, aunque fue ligeramente superior en las chicas sin haber ninguna con un patrón dietético pobre. Los hábitos alimenticios sobre la dieta mediterránea de los adolescentes en función del sexo y ciclo, teniendo en cuenta los 16 ítems se encuentran en la Tabla 2. 
Tabla 2. Frecuencia (Porcentaje) de respuestas afirmativas a ítems del Cuestionario sobre Adherencia a la Dieta Mediterránea y el total de la muestra dividida por $\operatorname{sexos}^{(*)}$

\begin{tabular}{|c|c|c|c|c|c|c|c|c|c|}
\hline & Chicas & & & Chicos & & & Total & & \\
\hline $\begin{array}{l}\text { Ítem Adherencia a Dieta } \\
\text { Mediterránea }\end{array}$ & \begin{tabular}{|l|} 
ler Ciclo \\
ESO
\end{tabular} & $\begin{array}{l}2^{\circ} \text { Ciclo } \\
\text { ESO }\end{array}$ & Total & \begin{tabular}{|l|} 
ler Ciclo \\
ESO
\end{tabular} & $\begin{array}{l}2^{\circ} \text { Ciclo } \\
\text { ESO }\end{array}$ & Total & \begin{tabular}{|l} 
ler Ciclo \\
ESO \\
\end{tabular} & $\begin{array}{l}2^{\circ} \text { Ciclo } \\
\text { ESO }\end{array}$ & Total \\
\hline Fruta todos los días & $56(17,6)$ & $40(81,6)$ & $96(82,1)$ & $41(75,9)$ & $26(68,4)$ & $67(72,8)$ & $97(79,5)$ & $66(75,9)$ & $163(78)$ \\
\hline $2^{\circ}$ Fruta & $30(44,1)$ & $20(40,8)$ & $50(42,7)$ & $22(40,7)$ & $12(31,6)$ & $34(37,0)$ & $52(42,6)$ & $32(36,8)$ & $84(40,2)$ \\
\hline Verdura 1/día & $39(57,4)$ & $16(32,7)$ & $55(47,0)$ & $27(50,0)$ & $18(47,4)$ & $45(48,9)$ & $66(54,1)$ & $34(39,1)$ & $100(47,8)$ \\
\hline Verdura +1/día & $14(20,6)$ & $8(16,3)$ & $22(18,8)$ & $11(20,4)$ & $6(15,8)$ & $17(18,5)$ & $25(20,5)$ & $14(16,1)$ & $39(18,7)$ \\
\hline Pescado 2-3 & $47(69,1)$ & $33(67,3)$ & $80(68,4)$ & $34(63,0)$ & $20(52,6)$ & $54(58,7)$ & $81(66,4)$ & $53(60,9)$ & $134(64,1)$ \\
\hline Hamburguesería & $21(30,9)$ & $18(36,7)$ & $39(33,3)$ & $15(27,8)$ & $7(18,4)$ & $22(23,9)$ & $36(29,5)$ & $25(28,7)$ & $61(29,2)$ \\
\hline Legumbres +1 & $49(72,1)$ & $36(73,5)$ & $85(72,6)$ & $36(66,7)$ & $25(65,8)$ & $61(66,3)$ & $85(69,7)$ & $61(70,1)$ & $146(69,9)$ \\
\hline Pasta/arroz +5 & $27(39,7)$ & $16(32,7)$ & $43(36,8)$ & $21(38,9)$ & $15(39,5)$ & $36(39,1)$ & $48(39,3)$ & $31(35,6)$ & $79(37,8)$ \\
\hline Desayuna cereal & $53(77,9)$ & $34(69,4)$ & $87(74,4)$ & $38(70,4)$ & $25(65,8)$ & $63(68,5)$ & $91(74,6)$ & $59(67,8)$ & $150(71,8)$ \\
\hline Frutos secos 2-3 & $33(48,5)$ & $23(46,9)$ & $56(47,9)$ & $30(55,6)$ & $19(50,0)$ & $49(53,3)$ & $63(51,6)$ & $42(48,3)$ & $105(50,2)$ \\
\hline Aceite oliva para cocinar & $67(98,5)$ & $48(98,0)$ & $115(98,3)$ & $49(90,7)$ & $34(89,5)$ & $83(90,2)$ & $116(95,1)$ & $82(94,3)$ & $198(94,7)$ \\
\hline No desayuno & $10(14,7)$ & $15(30,6)$ & $25(21,4)$ & $12(22,2)$ & $9(23,7)$ & $21(22,8)$ & $22(18,0)$ & $24(27,6)$ & $46(22)$ \\
\hline Desayuna lácteo & $51(75,0)$ & $37(75,5)$ & $88(75,2)$ & $33(61,1)$ & $29(76,3)$ & $62(67,4)$ & $84(68,9)$ & $66(75,9)$ & $150(71,8)$ \\
\hline Desayuna bollería & $15(22,1)$ & $16(32,7)$ & $31(26,5)$ & $17(31,5)$ & $12(31,6)$ & $29(31,5)$ & $32(26,2)$ & $28(32,2)$ & $60(28,7)$ \\
\hline 2 yogures/queso al día & $34(50)$ & $27(55,1)$ & $61(52,1)$ & $32(59,3)$ & $18(47,4)$ & $50(54,3)$ & $66(54,1)$ & $45(51,7)$ & $111(53,1)$ \\
\hline Varios dulces & $29(42,6)$ & $19(38,8)$ & $48(41,0)$ & $28(51,9)$ & $13(34,2)$ & $41(44,6)$ & $57(46,7)$ & $32(36,8)$ & $89(42,6)$ \\
\hline $\mathrm{N}$ & 68 & 49 & 117 & 54 & 38 & 92 & 122 & 87 & 209 \\
\hline Total $^{*}$ & $8,46(1,82)$ & $8,24(2,02)$ & $8,37(1,90)$ & $8,28(2,03)$ & $7,58(2,16)$ & $7,99(2,10)$ & $8,38(1,91)$ & $7,95(2,10)$ & $8,20(2)$ \\
\hline Índice KIDMED & & $\begin{array}{l}\text { Chicas } \\
\mathrm{N}(\%)\end{array}$ & & & $\begin{array}{l}\text { Chicos } \\
\mathrm{N}(\%)\end{array}$ & & & $\begin{array}{l}\text { Total } \\
\mathrm{N}(\%)\end{array}$ & \\
\hline $\begin{array}{l}\leq 3 \\
4-7 \\
\geq 8\end{array}$ & & $\begin{array}{l}0(0) \\
39(33,3) \\
78(66,7)\end{array}$ & & & $\begin{array}{l}1(1,1) \\
32(34,8) \\
59(64,1)\end{array}$ & & & $\begin{array}{l}1(0,5) \\
71(34,0) \\
137(65,6)\end{array}$ & \\
\hline
\end{tabular}

$\left({ }^{*}\right)$ El ítem 'Total' se corresponde a la puntuación final del cuestionario. Únicamente en este ítem los datos se muestran en valores promedio (desviación estándar).

En la tabla 3, se muestra el análisis de los ítems del cues- $\quad$ tionario IPAQ, según el Sexo y Etapa educativa.

Tabla 3. Descriptivos del Cuestionario Internacional de Actividad Física. Valor promedio (desviación estándar).

\begin{tabular}{|c|c|c|c|c|}
\hline & \multicolumn{2}{|c|}{ Chicas } & \multicolumn{2}{|c|}{ Chicos } \\
\hline & $1^{\text {er }}$ Ciclo ESO & $2^{\circ}$ Ciclo ESO & $1^{\text {er }}$ Ciclo ESO & $2^{\circ}$ Ciclo ESO \\
\hline Realizas actividad física &, $66(, 48)$ &, $51(, 51)$ & ,93(,26) &, $89(, 31)$ \\
\hline AF o Deporte & $1,25(, 92)$ & ,90(,94) & $1,35(, 65)$ & $1,34(, 67)$ \\
\hline Veces por semana & $1,76(1,42)$ & $1,53(1,68)$ & $2,74(1,22)$ & $3,00(1,43)$ \\
\hline Actividad física pesada & $1,60(1,57)$ & $1,43(1,93)$ & $2,76(2,27)$ & $2,68(2,14)$ \\
\hline Actividad física intensa & $61,76(68,54)$ & $46,53(76,25)$ & $74,81(100,99)$ & $67,18(65,14)$ \\
\hline Actividad física moderada & $1,65(1,61)$ & $1,84(2,03)$ & $2,39(1,99)$ & $2,50(1,69)$ \\
\hline Tiempo AF moderada & $48,24(53,61)$ & $40,00(48,73)$ & $59,81(63,04)$ & $75,26(83,66)$ \\
\hline Caminar $10 \mathrm{~min}$ & $3,66(2,52)$ & $4,43(2,31)$ & $3,91(2,66)$ & $4,61(2,03)$ \\
\hline Tiempo camina & $46,99(45,10)$ & $37,04(25,82)$ & $65,00(112,98)$ & $50,13(37,41)$ \\
\hline Sentado & $268,68(167,64)$ & $383,67(164,50)$ & $235,56(158,65)$ & $256,63(163,74)$ \\
\hline Caminata total (METS) & $705,37(861,74)$ & $605,11(481,12)$ & $1056,61(2276,48)$ & $807,63(768,88)$ \\
\hline
\end{tabular}




\begin{tabular}{lllll}
\hline & \multicolumn{2}{l}{ Chicas } & \multicolumn{2}{c}{ Chicos } \\
\cline { 2 - 5 } & $1^{\text {er }}$ Ciclo ESO & $2^{\text {o } C i c l o ~ E S O ~}$ & $1^{\text {er }}$ Ciclo ESO & $2^{\circ}$ Ciclo ESO \\
\hline Moderada (METS) & $583,53(897,90)$ & $605,71(918,04)$ & $823,70(1231,96)$ & $1140,00(2182,72)$ \\
Vigorosa (METS) & $1429,41(2106,78)$ & $1484,08(3681,96)$ & $2256,30(3678,01)$ & $2049,47(2258,71)$ \\
TOTAL & $1557,58(1282,75)$ & $1594,50(1087,25)$ & $4136,61(5266,82)$ & $3997,11(38663,75)$ \\
MIN/DIA & $156,99(116,23)$ & $123,57(115,70)$ & $199,63(198,21)$ & $192,58(120,13)$ \\
\hline
\end{tabular}

El estadístico de Kruskal Wallis no mostró diferencias significativas entre los valores de los rangos promedios de las variables IPAQ $(H(5)=, 807, p=, 977)$, pero si entre los valores de los rangos promedio de DM $(H(5)=13,870, p=, 016)$, según los distintos niveles de la variable IMC (véase Figuras 1 y 2 ).

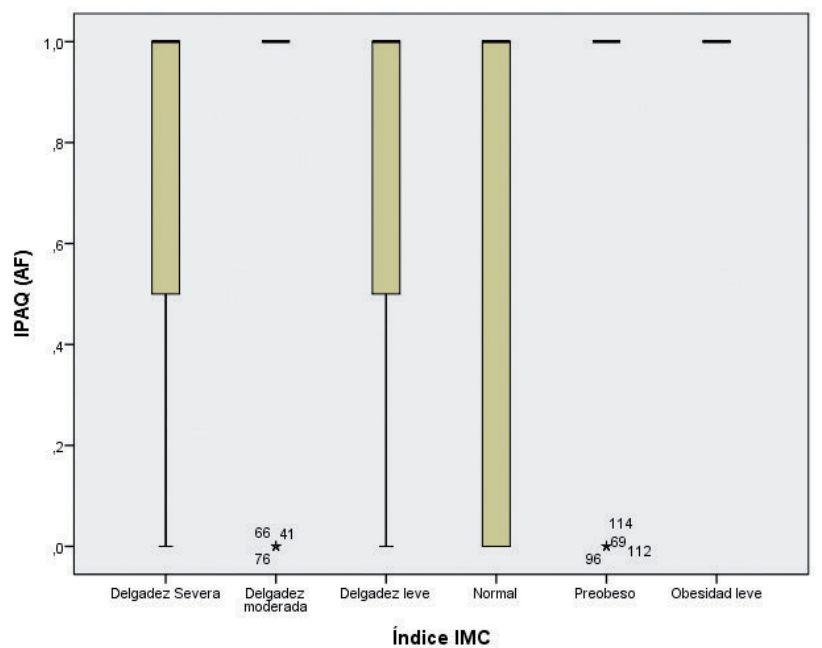

Figura 1. Diferencias observadas entre los valores de rangos promedios del nivel de actividad física según los distintos niveles del Índice de Masa Corporal.

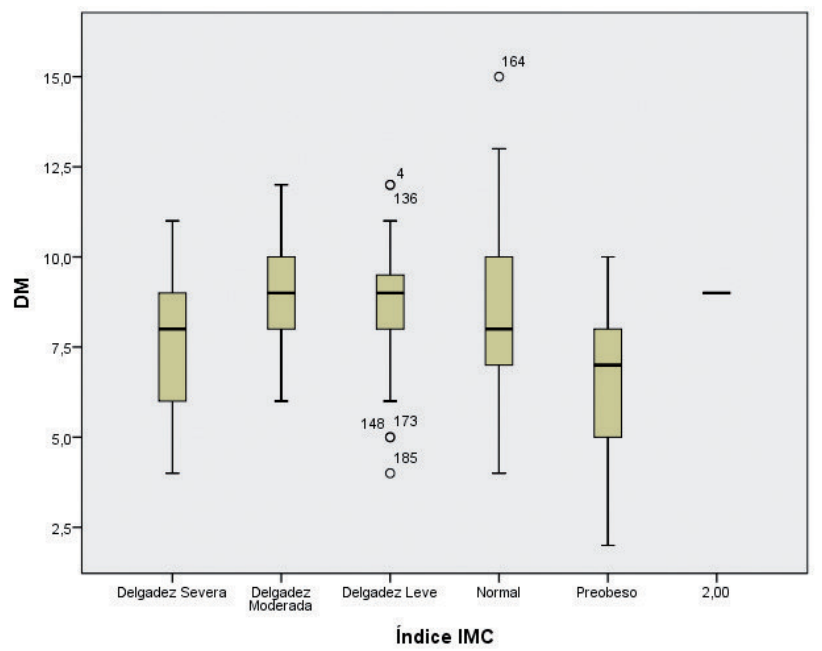

Figura 2. Diferencias observadas entre los valores de rangos promedios del nivel de adherencia a la dieta mediterránea, según los distintos niveles del Índice de Masa Corporal.

SPORT TK: Revista Euroamericana de Ciencias del Deporte

ISSN edición web: 2340-8812 / vol. 7, n.o 2 (Supl. 1) / Murcia / Julio 2018 / Págs. 43-52
En cuanto al Sexo, el estadístico U de Mann Whitney para muestras independientes arrojó diferencias estadísticamente significativas entre los valores de rangos promedio de IPAQ $(U=3688, p=, 000, r=0,40)$ presentando las mujeres rangos promedio significativamente inferiores a los hombres (90,52 y 123,41 respectivamente). Por el contrario, no se observan diferencias inter-sexos en la variable Adherencia a la $\mathrm{DM}(U=4726, p=, 126, r=0,12)$ (Figuras 3 y 4$)$.

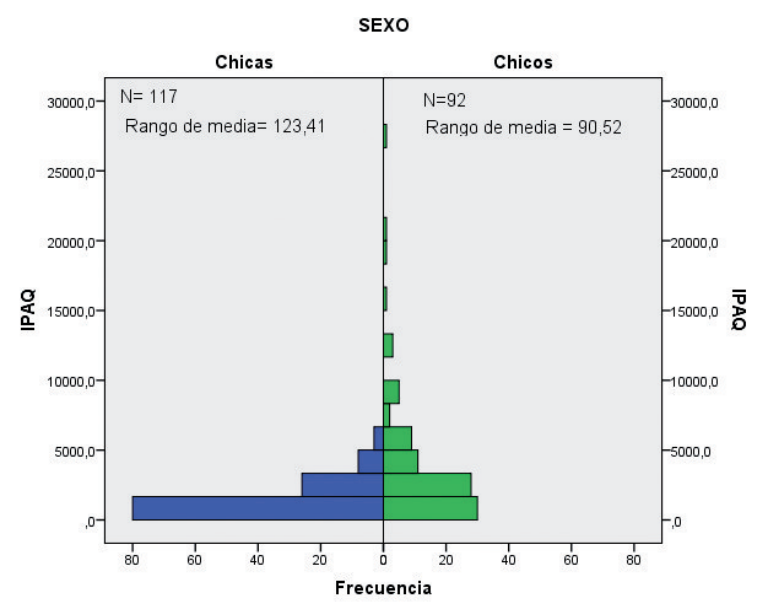

Figura 3. Rangos promedio de IPAQ según el sexo de los participantes. Diferencias mostradas por la prueba U de Mann Whitney para muestras independientes.

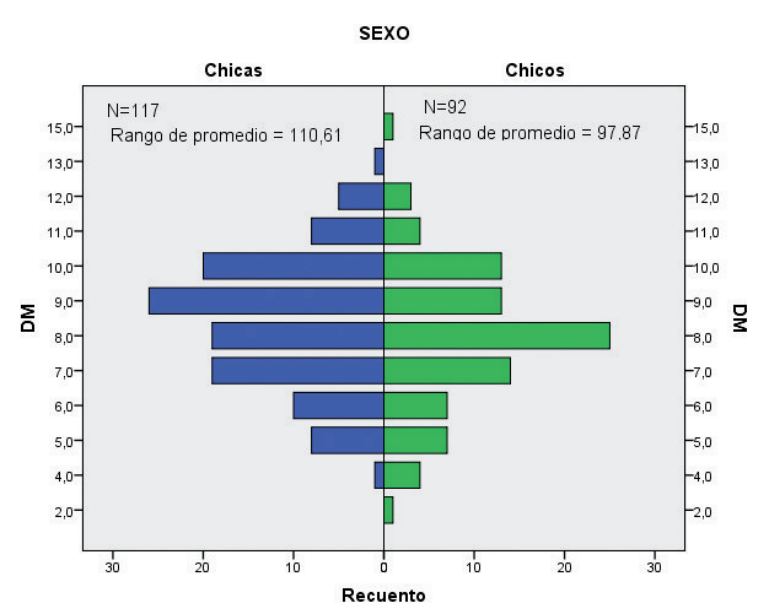

Figura 4. Rangos promedio de DM según el sexo de los participantes. Diferencias mostradas por la prueba U de Mann Whitney para muestras independientes. 
El análisis por parejas realizado mediante la prueba $U$ de Mann Whitney no destacó diferencias significativas entre el par Primer ciclo de ESO - Segundo ciclo de ESO (Véase tabla 4).

Tabla 4. Comparación por pares según la Etapa educativa respecto al Nivel de Actividad Física.

\begin{tabular}{|c|c|c|c|c|c|}
\hline Muestra 1 - Muestra 2 & Estadístico de prueba & Estándar error & Desv. Estadístico de prueba & Sig. & Sig. ajuste \\
\hline $1^{\text {er }}$ Ciclo ESO $-2^{\circ}$ Ciclo ESO & $-12,173$ & 10,026 & $-1,214$ &, 225 & ,674 \\
\hline
\end{tabular}

Por otro lado, teniendo en cuenta las comparaciones entre los pares que se constituyen a partir de los niveles de la variable Etapa educativa, según cada sexo, sobre los valores de ran- gos promedio de DM e IPAQ (véase Tabla 5) no se detectan diferencias estadísticamente significativas en IPAQ.

Tabla 5. Comparación entre pares de los distintos niveles de Etapa educativa según el Sexo, sobre Adherencia a la dieta mediterránea y Nivel de Actividad Física. Prueba U de Mann Whitney.

\begin{tabular}{lllll}
\hline & Chicas & \multicolumn{3}{l}{ Chicos } \\
\hline & DM & IPAQ & DM & IPAQ \\
\hline $1^{\text {er }}$ Ciclo ESO- $2^{\circ}$ Ciclo ESO & $U=1560,0 p=, 553$ & $U=1821,5 p=, 859$ & $U=889,5 p=, 272$ & $U=1153,2 p=, 312$ \\
\hline
\end{tabular}

Finalmente, de acuerdo a los resultados de la Tabla 6, el estadístico $\mathrm{R}$ de Spearman mostró una relación estadísticamente significativa, de signo positivo, entre los pares de vari- ables IPAQ-Edad ( $\mathrm{P}=0,001)$, e IMC-Edad $(\mathrm{P}=, 000)$. Este comportamiento fue similar, diferenciando entre sexos.

Tabla 6. Análisis de correlación. Estadístico R de Spearman.

\begin{tabular}{|c|c|c|c|c|c|}
\hline & & IPAQ & Dieta Mediterránea & IMC & Edad \\
\hline \multirow[t]{3}{*}{ IPAQ } & Coeficiente de correlación & 1,000 & ,086 & ,108 &,- 019 \\
\hline & Sig. (bilateral) & . & ,217 & 089 & ,784 \\
\hline & $\mathrm{N}$ & 209 & 209 & 209 & 209 \\
\hline \multirow[t]{3}{*}{ Dieta Mediterránea } & Coeficiente de correlación & & 1,000 &,- 127 &,- 069 \\
\hline & Sig. (bilateral) & & . & 068 & ,324 \\
\hline & $\mathrm{N}$ & & 209 & 209 & 209 \\
\hline \multirow[t]{3}{*}{ IMC } & Coeficiente de correlación & & & 1,000 &, $416^{* *}$ \\
\hline & Sig. (bilateral) & & & . &, 000 \\
\hline & $\mathrm{N}$ & & & 209 & 209 \\
\hline \multirow[t]{3}{*}{ Edad } & Coeficiente de correlación & & & & 1,000 \\
\hline & Sig. (bilateral) & & & & . \\
\hline & $\mathrm{N}$ & & & & 209 \\
\hline
\end{tabular}

\section{Discusión}

Uno de los principales hallazgos encontrados en este estudio es que el total de los estudiantes participantes fueron normo-peso con un IMC saludable, no existiendo ninguno con sobrepeso ni obesidad. Estos resultados coinciden con otros estudios que indicaron que la mayoría de los adolescentes espańoles se encuentra dentro de la categoría de normo-peso (Cabrero, García, Salinero, Pérez, Fernández \& García, 2012;
Doménech et al., 2015; San Mauro et al., 2015). Sin embargo, discrepan de los porcentajes en adolescentes de 12 a 18 años, donde se apreció sobrepeso en un 18,8 \% y obesidad en un 29,3\% (Navarro-Solera, Gónzalez-Carrascosa \& Soriano, 2014). Resultados similares, reportaron Doménech et al. (2015), con escolares de 12 y 16 años concluyendo que más del $14,6 \%$ en chicos y $4,2 \%$ en chicas de esos escolares adolescentes padecían sobrepeso.

En nuestro estudio existió diferencias estadísticamente 
significativas en el peso y talla según el sexo, no existiendo diferencias en los valores del IMC, datos en consonancia con los obtenidos en niños y adolescentes de 6 a 16 años (San Mauro et al., 2015).

Igualmente se observó una relación de signo positivo entre el IMC y Edad (Tabla 6). Investigaciones confirman la asociación positiva significativa existente entre el aumento gradual del IMC y el avance de la edad cronológica, como una evolución natural del IMC (Garagorri, 2004).

En cuanto a la DM más de la mitad de la muestra evaluada presentó una adherencia alta, con un porcentaje del 66,7\% en las chicas y un $64,1 \%$, en los chicos sin diferencias significativas. Sólo el $0,5 \%$ de adolescentes de nuestra muestra reflejaron una baja adherencia a la DM y el 34\% restante presentaron una adherencia media y, por tanto, necesitarían mejorar su patrón al prototipo mediterráneo. Nuestros resultados coinciden con los reportados en escolares espańoles rurales que confirman hábitos alimentarios más mediterráneos que los residentes en localidades urbanas (Ayechu \& Durá, 2009). No obstante, son mayores que los obtenidos en diversos estudios con adolescentes españoles. En función del sexo, las chicas presentan una alta adhesión al patrón mediterráneo $(68,4 \%)$, siendo este porcentaje más alto, con respecto a los $52,8 \%$ en valencianas de la ESO (Navarro-Solera et al., 2014) a los 46,9\% en granadinas de 10-16 años (Mariscal-Arcas, Rivas, Velasco, Ortega, Caballero \& Olea-Serrano, 2009) a los 42,9\% en adolescentes de 13-16 años pamploneses (Ayechu y Durá, 2009) a los 37,1\% de las chicas de secundaria de Leganés (Rodríguez et al., 2012), y muy superior a los 31,3\% de los escolares de ESO de la Comunidad de Madrid (Zapico, Blández \& Fernández, 2010), y a los 24,3\% de las adolescentes canarias (López, Navarro, Ojeda, Brito, Ruiz \& Navarro, 2013).

La puntuación media del KIDMED fue ligeramente superior en las chicas que en los chicos $(8,37 \pm 1,90$ vs $7,99 \pm 2,10$ respectivamente) sin diferencias significativas entre sexos ni ciclo educativo (Tabla 2). Estos resultados no corroboran la tendencia de un deterioro progresivo de la adherencia a la DM en relación a la edad como en Grao-Cruces et al. (2013), quienes en su estudio con adolescentes de 11 a 18 años del sur de Espańa manifestaron que la adherencia fue menor en los adolescentes de mayor edad $(\mathrm{P}<0,001)$, sin diferir entre sexos.

$\mathrm{Al}$ analizar las respuestas del KidMed, sólo existió diferencias significativas en el ítem "utilizan aceite de oliva para cocinar en casa” donde el $98,3 \%$ de las chicas contestaron afirmativamente frente al $90,2 \%$ de los chicos existiendo diferencias entre ambos sexos. En general, como aspectos positivos, destacar un consumo importante de una pieza de fruta todos los días (78,0\%); los lácteos en el desayuno ( $75,2 \%$ vs $67,4 \%$ para chicas y chicos respectivamente) el consumo de legumbres a la semana $(72,6 \%$ chicas y $66,3 \%$ chicos), la ingesta de cereales en el desayuno $(74,4 \%$ chicas vs $68,5 \%$ chicos) y siendo aceptable también el consumo $2-3$ veces a la semana de una pieza de pescado $(68,4 \%$ en chicas y $58,7 \%$ en chicos. Por otro lado, se obtuvieron porcentajes importantes en ciertas características negativas como tomar golosinas varias veces al día $(41,0 \%$ en chicas y $44,6 \%$ en chicos); acudir semanalmente a restaurantes de comida rápi$\mathrm{da}(33,3 \%$ y $23,9 \%$ respectivamente. y no desayunar $(21,4 \%$ en las chicas y $22,8 \%$ en los chicos). Estos datos indican, que aunque el consumo de frutas, legumbres y pescado es suficiente en nuestra muestra, sin embargo existe un desequilibrio en su dieta, debido al gran consumo de dulces o golosinas por ser alimentos ricos en azúcares sencillos y grasas hidrogenadas; a la realización de comidas rápidas las cuales ofrecen alimentos de alta densidad calórica y al hecho de no desayunar siendo ésta una de las comidas más importantes del día, directamente implicada en el mantenimiento de una actividad física e intelectual durante la mañana.

No se encontraron diferencia significativas entre la adherencia a la DM y el IMC en función del sexo, ni ciclo escolar, resultados coincidentes con los adolescentes rurales españoles (Grao-Cruces et al., 2013). Sin embargo, si se evidenció diferencias estadísticamente significativas entre los valores promedio de la DM con respecto al sexo, datos que corrobora el estudio de Cabrero et al. (2012), siendo en este caso mejor en los chicos que en las chicas.

Respecto a la AF, gran número de adolescentes dedicaron un tiempo elevado a la práctica de actividad física. Los resultados por grupos de género mostraron un mayor nivel de AF en los chicos respecto a las chicas, con diferencias estadísticamente $(\mathrm{P}<0,001)$. Estos datos son similares a los de Martínez et al. (2012), en adolescentes con un rango de edad igual a nuestro estudio, pero no son coincidentes con autores quienes indican que con el incremento de la edad en los adolescentes, se disminuye la motivación hacia la práctica de AF y como consecuencia, una reducción de la mismas (Gil, Cuevas \& Contreras 2012; Martínez et al., 2012).

En relación a las recomendaciones de actividad física ( $\geq 60$ $\mathrm{min} /$ día de actividad física de moderada a vigorosa) el 84,7\% alcanzaron el limite recomendado, hallazgo no coincidente con Mielgo-Ayuso et al. (2016), donde solo el 36,2\% de la muestra cumplió con dichas recomendaciones. En nuestro estudio el porcentaje de adolescentes que cumple con las recomendaciones de AF es mayor en chicos que en chicas $(93,5 \%$ vs $76,3 \%$ ), resultados consistentes con otros estudios donde los chicos igualmente realizan más AF que las chicas y siendo ésta más vigorosa en chicos que en chicas (Baena-Extremera \& Granero-Gallegos, 2015; San Mauro et al., 2015).

En cuanto a la asociación entre AF e IMC nuestros resultados no revelaron relaciones significativas entre la muestra total ni en función del sexo, ni ciclo escolar.

Como principales limitaciones de nuestro estudio, desta- 
car la escasa muestra representativa de la población general por ser datos de sólo dos institutos rurales, con lo cual, la generalización de los resultados deben realizarse con cautela. Igualmente, la utilización de un test indirecto como el IPAQ para la valoración de la AF, no obstante, se puede indicar que se trata de un cuestionario que ha demostrado su validez y fiabilidad en 12 países y en contextos variados (Wareham, Jakes, Rennie, Mitchell, hennings \& Day, 2002) Por último, datos adicionales como la condición socioeconómica no fueron valorados los cuales podrían afectar al grado de adherencia a la DM y a la práctica de AF en los adolescentes de nuestro estudio.

\section{Conclusiones}

Un alto porcentaje de la muestra mostró óptima adherencia a la dieta mediterránea (65,6\%). El valor medio del IMC de los adolescentes indicó normalidad nutricional sin diferencias entre sexos ni ciclo escolar. Una proporción importante de los adolescentes cumplieron con las recomendaciones de AF $(76,3 \%$ y $93,5 \%$ chicas y chicos respectivamente). Los chicos mostraron mayor nivel de actividad física que las chicas, existiendo diferencias significativas entre ambos. No se encontraron asociaciones entre DM, AF, IMC y género. Las únicas relaciones significativas de signo positivo fueron entre los pares IMC-Edad.

Sugerimos para futuros estudios, incrementar la muestra a otros cursos tanto de bachillerato como de educación primaria, así como, a otros pueblos de diferentes zonas de Granada y provincias de Andalucía para poder obtener datos con mayor valor estadístico y llevar a efecto una comparación entre cursos y provincias. Igualmente, se podría comprobar el nivel de AF con otro cuestionario como el Global Physical Activity Questionnaire y con métodos directos utilizando acelerómetros, realizando así una comparación con métodos indirectos. Finalmente, sería conveniente comprobar la evolución de las variables analizadas mediante estudios longitudinales.

\section{Referencias bibliográficas}

1. Acosta, M.V., Llopis, J.M., Gómez, G. \& Pineda, G. (2005). Evaluación de la conducta alimentaria de riesgo. Estudio transcultural entre adolescentes de España y México. International Journal of Psychology and Psychological Therapy, 5(3), 223-232.

2. Ayechu, A. y Durá, T. (2009). Dieta mediterránea y adolescentes. $N u$ trición Hospitalaria, 24(6), 751-762.

3. Baena-Extremera, A. \& Granero-Gallegos, A. (2015). Educación física e intención de práctica física en tiempo libre. Revista Electrónica de Investigación Educativa, 17(3), 132-144.

4. Balaguer, I. (2004). Control y prevención de las enfermedades cardiovasculares en el mundo. Revista Española de Cardiología, 57(6), 487-94.

5. Blaes, A., Baquet, G., Fabre, C., Van Praagh, E. \& Berthoin, S. (2011). Is there any relationship between physical activity level and patterns, and physical performance in children. International Journal of Behavioral Nutrition and Physical Activity, 8(1), 122.

6. Cabrero, M., García, A., Salinero, J., Pérez, B., Fernández, J. \& García, R. (2012). Diet quality and its relation to sex and BMI adolescents. Revista Española de Nutrición Clínica y Dietética Hospitalaria, 32(2), 21-27.

7. Cole, T., Flegal, K., Nicholls, D. \& Jackson, A. (2007). Body mass index cut off sto define thinness in children and adolescents. International Survey, 335, 194-197.

8. Doménech, G., Sánchez, Á. \& Ros, G. (2015). Estudio transversal para evaluar los factores asociados a las diferencias entre la ciudad y los distritos de estudiantes de la escuela secundaria del sureste de Espańa ( Murcia ) para su adhesión a la dieta mediterránea. Nutrición Hospitalaria, 31(3), 1359-1364.

9. Garagorri, J. M. (2004). Hipercrecimientos. Sistema diagnóstico. Anales Españoles de Pediatría, 60(4), 291-295.

10. García, G. Garrido, A. \& Ojembarrena, M. (2008). Recomendaciones de dieta y ejercicio en niños y adolescentes.

11. García, S., Herrera, N., Rodríguez, C., Nissensohn, M., Román-Viñas, B. \& Serra-Majem, L. (2015). KIDMED test ; prevalence of low adherence to the Mediterranean Diet in children and young; a systematic review. Nutrición Hospitalaria, 32(6), 2390-2399.

12. Gil, P., Cuevas, R. \& Contreras, O. (2012). Educación física y hábitos de vida activa: percepciones de los adolescentes y relación con el abandono deportivo. Aula Abierta, 40(3), 115-124.

13. Grao-Cruces, A., Nuviala, A., Fernandez-Martinez, A., Porcel- Galvez, A., Moral-Garcia, J. \& Martinez-Lopez, E. (2013). Adherence to the mediterranean diet in rural and urban adolescents of southern Spain, life satisfaction, anthropometry, and physical and sedentary activities. Nutrición Hospitalaria, 28(4), 1129-1133.

14. HealthyChildren.org. (2017). Etapas de la adolescencia. Retrieved from https:/www.healthychildren.org/Spanish/ages-stages/teen/Paginas/ Stages-of-Adolescence.aspx [Accessed 19 Jan. 2017].

15. Labrado, S. (2011). Diferencias de género niveles de práctica de actividad física y hábitos saludables en la población adolescente de Castilla-La Mancha Eficacia de un Programa de Intervenciónen los.

16. López, E., Navarro, M., Ojeda, R., Brito, E., Ruiz, J. A. \& Navarro, M (2013). Adecuación a la dieta mediterránea y actividad física en adolescentes de Canarias. Archivos de Medicina Del Deporte, 30, 208-14.

17. Mariscal-Arcas, M., Rivas, A., Velasco, J., Ortega, M., Caballero, A M. \& Olea-Serrano, F. (2009). Evaluation of the Mediterranean Diet Quality Index (KIDMED) in children and adolescents in Southern Spain. Public Health Nutrition, 12(9), 1408-1412.

18. Martínez-González, M. Á., De la Fuente-Arrillaga, C., Nunez-Cordoba, J. M., Basterra-Gortari, F. J., Beunza, J. J., Vazquez, Z., \& BesRastrollo, M. (2008). Adherence to Mediterranean diet and risk of developing diabetes : prospective cohort study. British Medical Journal, 336(7657), 1348-1351.

19. Martínez, A., Chillón, P., Martín-Matillas, M., Pérez, I., Castillo, R., Zapatera, B...\& Delgado, M. (2012). Motivos de abandono y no práctica de actividad físico-deportiva en adolescentes espańoles: estudio Avena. Cuadernos de Psicología del Deporte.

20. Mielgo-Ayuso, J., Aparicio-Ugarriza, R., Castillo, A., Ruiz, E., Ávila, J. M., Aranceta-Batrina, J., \& González-Gross, M. (2016). Physical activity patterns of the Spanish population are mostly determined by sex and age: findings in the ANIBES Study. PloS One, 11(2).

21. Muñoz, S. (2005). La educación física escolar ante el problema de la obesidad y el sobrepeso. Revista Internacional de Medicina y Ciencias de La Actividad Física y Del Deporte, 5(19), 179-199. 
22. Navarro-Solera, M., Gónzalez-Carrascosa, R. \& Soriano, J. M. (2014). Estudio del estado nutricional de estudiantes de educación primaria y secundaria de la provincia de Valencia y su relación con la adherencia a la Dieta Mediterránea. Revista Española de Nutrición Humana Y Dietética, 18(2), 81-88.

23. Rodríguez, M., García, A., Salinero, JJ., Pérez, B., Sánchez, J.J., García, R. \& Ibańez, R. (2012). Calidad de la dieta y su relación con el IM y el sexo en adolescentes. Nutrición Clínica y Dietética Hospitalaria, 32(2), 21-27.

24. San Mauro, I., Megías, A., García de Angulo, B., Bodega, P., Rodríguez, P., Grande, G... \& Gariciano, E. (2015). Influencia de hábitos saludables en el estado ponderal de nińos y adolescentes en edad escolar. Nutrición Hospitalaria, 31(5), 1996-2005.

25. Sánchez-Cruz, J-J., Jiménez-Moleón, J.J., Fernández-Quesada, F. \& Sánchez, M. J. (2012). “ a en 2012 Prevalencia de obesidad infantil y juvenil en Espan. Revista Española de Cardiología, 66(5), 371-376.

26. Sánchez-Cruz, J. J., Jiménez-Moleón, J. J., Fernández-Quesada, F., \& Sánchez, M. J. (. (2013). Prevalencia de obesidad infantil y juvenil en
España en 2012. Revista Española de Cardiología, 66(5), 371-376.

27. Serra-Majem, L., Ribas, L., Ngo, J., Ortega, R., García, A., Pérez-Rodrigo, C. \& Aranceta, J. (2004). Food, youth and the Mediterranean diet in Spain . Development of KIDMED, Mediterranean Diet Quality Index in children and adolescents. Public Health Nutrition, 7(7), 931-935.

28. Tercedor, P., Martín-Matillas, M., Chillón, P., Pérez-López, I., Ortega, F., \& Wärnberg, J. (2007). Increase in cigarette smoking and decrease in the level of physical activity among Spanish adolescents. AVENA study. Nutricion Hospitalaria, 22(1), 89-94.

29. Valera-Moreinas, G. (2013). Libro Blanco de Nutrición en España. Madrid: Fundación Española de Nutrición (FEN)

30. Wareham, N., Jakes, R., Rennie, K., Mitchell, J., Hennings, S., \& Day, N. (2002). alidity and repeatability of the EPIC-Norfolk physical activity questionnaire. International Journal of Epidemiology, 31(1), 168-74.

31. Zapico, A., Blández, J. \& Fernández, E. (2010). Overweight, obesity and adequacy to mediterranean diet of Madrid Community adolescents. Archivos de Medicina del Deporte, 27(138), 271-280. 